\title{
Tissue hyaluronan expression, as reflected in the sputum of lung cancer patients, is an indicator of malignancy
}

\begin{abstract}
Hyaluronan (HA) shows promise for detecting cancerous change in pleural effusion and urine. However, there is uncertainty about the localization of $\mathrm{HA}$ in tumor tissue and its relationship with different histological types and other components of the extracellular matrix, such as angiogenesis. We evaluated the association between HA and degree of malignancy through expression in lung tumor tissue and sputum. Tumoral tissue had significantly increased HA compared to normal tissue. Strong HA staining intensity associated with cancer cells was significant in squamous cell carcinoma compared to adenocarcinoma and large cell carcinoma. A significant direct association was found between tumors with a high percentage of HA and MVD (microvessel density) in tumoral stroma. Similarly significant was the direct association between N1 tumors and high levels of HA in cancer cells. Cox multivariate analysis showed significant association between better survival and low HA. HA increased in sputum from lung cancer patients compared to cancer-free and healthy volunteers and a significant correlation was found between HA in sputum and HA in cancer tissue. Localization of HA in tumor tissue was related to malignancy and reflected in sputum, making this an emerging factor for an important diagnostic procedure in patients suspected to have lung cancer. Further study in additional patients in a randomized prospective trial is required to finalize these results and to validate our quantitative assessment of $\mathrm{HA}$, as well as to couple it to gold standard sputum cytology.
\end{abstract}

Key words: Hyaluronic acid; Sputum; Lung cancer; Immunohistochemistry; ELISA

\section{Introduction}

Lung cancer (LC) is the major cause of death among other types of cancer in the world (1). Since the last record, LC represented 224,210 new cases with 159,260 deaths according to SEER Cancer Statistic Review (2). Another concerning fact about LC is the mortality rate, which is responsible for 75.2 deaths/100,000 men and 52.3 deaths/ 100,000 women in the United States (2). In Brazil, the National Institute of Cancer (INCA) estimated 27,330 new cases of LC in 2014 (http://www.inca.gov.br/estimativa/2014/).
The high mortality rates are closely related to the diagnosis that is usually performed in the later stages of the disease when the treatment options are only palliative (3). The high incidence and mortality of patients with LC indicate urgency for new diagnostic procedures and prognostic factors for early detection of recurrence and shortening of a patient's life. In this context, sputum is an attractive potential source of biomarkers for LC because it can be obtained noninvasively and may represent the field of

Correspondence: V.L. Capelozzi: <vcapelozzi@lim05.fm.usp.br>. 
injury. Consequently, many research groups have been examining sputum for potential biomarkers, but, to date, there are no definitive data indicating that sputum biomarkers are sufficiently sensitive and specific to reliably predict which high-risk patients will go on to develop LC.

Thus, our group studied molecular or other markers in the primary tumor as well as the stroma tissue milieu to determine what might relate to tumor recurrence and shortened survival (4-6). Because cell-extracellular matrix interactions participate in several steps required for tumor cell invasion and formation of metastases, a group of glycosaminoglycans has been targeted as a potentially useful tumor marker $(4,5)$. Among these glycosaminoglycans, hyaluronan (HA) has shown promise. HA is a linear polysaccharide chain, composed of repeating disaccharide units of $\mathrm{N}$-acetyl-D-glucosamine-b $(1,4)$-D-glucuronic acid-b $(7,8)$, which exists in both a high molecular mass form (1-66106 Da) and a polydisperse lower molecular mass form $(0.1-0.56106 \mathrm{Da})$, with the latter predominating under neoplastic conditions (9). HA has diverse biological functions in migration and proliferation (10), cell growth, and differentiation (11), as well as in disease progression (12). Polymerization of $H A$ is regulated by the action of one or more of three synthases (HAS1, HAS2, and HAS3) (13) and the hyaluronidases (HYAL1 and HYAL2), present in various tissues including the lung, are responsible for its degradation (14). Degradation products of HA may enhance tumor growth by stimulating malignant neovascularization (15). A biotinylated HA probe has shown more sensitivity than gold standard cytology to detect malignant changes in pleural effusion of mesothelioma (16-20) and urine from patients with urologic cancer (21-24), with an impact on prognosis. In some studies, staining for HA in formalin-fixed paraffin-embedded (FFPE) tissue has also been found to be significantly associated with survival (25-29), but there has been uncertainty about localization of $\mathrm{HA}$ in tumor tissue and its relationship with different histological types and other components of the extracellular matrix, such as angiogenesis, as well as impact of malignancy on prognosis. More important, to our knowledge HA has never been evaluated in the sputum from LC patients.

In the light of previous data, the first step of this work was to evaluate qualitative and quantitative distribution of $\mathrm{HA}$ in FFPE tumor tissue and its relationship with histological types and angiogenesis as indicators of malignancy. Hypothesizing an impact of these indicators on prognosis of patients, in a second step we evaluated the reflection of these changes in frozen sputum and cancer tissue specimens. We found that HA expression in tissue is an indicator of malignancy and has a relationship with sputum from patients with LC.

\section{Patients and Methods}

\section{Sputum collection}

This study was approved by the Institutional Review Board of the Hospital das Clínias, Faculdade de Medicina,
Universidade de São Paulo (\#256/10). Spontaneous sputum was collected from 115 consecutive patients (mean age $63 \pm 10$ years; 76 male, 39 female), just prior to bronchoscopy, attending the Instituto do Cancer do Estado de São Paulo and the Hospital do Cancer de Barretos for suspected LC. Informed consent to provide a sputum sample was obtained from each patient at a previous clinical appointment. LC was subsequently confirmed through final clinical diagnosis in 90 patients (mean age 61; 59 male, 31 female) whose sputum was included in this study. Table 1 summarizes the clinical features of these 90 LC patients. They included 37 current smokers (median packs/year $=48$ ), 38 ex-smokers (median packs/year $=39$ ), and 15 never smokers. The final stages (tumor, node, metastasis: TNM) for these patients were T1 $(n=8)$, T2 $(n=24)$, T3 $(n=22)$, T4 $(n=36)$, N0 $(n=14), \quad N 1 \quad(n=76), \quad M 0 \quad(n=46)$, and M1 $(n=44)$. Histological diagnosis included 32 squamous cell carcinoma (SqCC), 56 adenocarcinoma (AD), and 2 large cell carcinoma (LCC). The median follow-up for these patients was 27 months, and 32 patients were censored for survival analysis at the last time of follow-up.

\section{Sputum induction}

Sputum was also collected from 25 patients classified as cancer-free and 15 as healthy volunteers. All 15 healthy volunteers, selected during an investigation for non-pulmonary diseases, such as ophthalmology or endocrinology, and the patients selected underwent sputum induction by 3 inhalations of $4 \%$ hypertonic saline for $7 \mathrm{~min}$ each (30). The sputum samples were stored on ice during collection, and were macroscopically separated from the saliva in sterile petri dishes with the help of a needle. After separation, the sputum samples were stored at $-80^{\circ} \mathrm{C}$ until $\mathrm{HA}$ analysis.

The sputum samples were thawed and incubated with $7 \mathrm{M}$ urea at $60^{\circ} \mathrm{C}$, until the complete breakdown of the sputum's hydrophobic associations. The protein concentration of each sample was measured using a NanoDrop 2000c (Thermo Scientific, USA). After protein concentration analysis, the samples were incubated with a proteolytic enzyme for $18 \mathrm{~h}$ at $60^{\circ} \mathrm{C}$, and $\mathrm{HA}$ chains were diluted (1:100) in blocking buffer [0.05 M Tris-HCl, $\mathrm{pH} 7.4$, $1 \%$ bovine serum albumin (BSA)].

\section{Frozen lung tissue}

Forty-six patients (mean age $65 ; 25$ male, 17 female) of the 90 with confirmed LC were clinically staged as T1$4 \mathrm{~N} 1 \mathrm{M} 0$ and were considered to have tumors potentially curable by surgical resection. These 46 lung specimens were included in the study for biochemical analysis, histological hematoxylin and eosin staining, and immunohistochemical and immunofluorescence analyses. Clinical staging used routine chest X-ray, bronchoscopy, computerized tomography of thorax and upper abdomen, abdominal ultrasound, and bone scan. Mediastinoscopy 
Table 1. Clinical features of lung cancer patients.

\begin{tabular}{lcc}
\hline & Sputum analysis $(\mathrm{n}=90)$ & Tissue analysis $(\mathrm{n}=46)$ \\
\hline Age & $61(37-89)$ & $65(44-84)$ \\
Gender (M/F) & $59 / 31$ & $25 / 17$ \\
Smoking history & & \\
$\quad$ Yes & 37 & 25 \\
No & 15 & 15 \\
$\quad$ Ex-smoker & 38 & 6 \\
Stage T & & \\
1 & 8 & 11 \\
2 & 24 & 24 \\
3 & 22 & 7 \\
4 & 36 & 4 \\
Stage N & & 25 \\
$\quad 0$ & 14 & 21 \\
1 & 76 & 46 \\
Stage M & & 0 \\
$\quad 0$ & 46 & 13 \\
1 & 44 & 27 \\
Histologic type & & 6 \\
Squamous cell carcinoma & 32 & 32 \\
Adenocarcinoma & 56 & \\
Large cell carcinoma & 2 & \\
Follow-up (months) & $(7-88)$ & \\
Patients censored for survival analysis & 32 & \\
at last time of follow-up & & \\
\hline
\end{tabular}

Data are reported as number or median and range.

and lymph node biopsy were additionally performed on patients whose lymph nodes had a short axis diameter of $>1 \mathrm{~cm}$. They included 25 current smokers (median packs/year $=48), 6$ ex-smokers (median packs/year $=39$ ), and 15 never smokers. The final pathological stages (TNM) of these patients included T1 $(n=11)$, T2 $(n=24)$, T3 $(n=7)$, T4 $(n=4), N 0(n=25), N 1(n=21), M 0(n=46)$, and M1 $(n=0)$. The tumors were classified as SqCC $(n=13), \operatorname{AD}(n=27)$, and LCC $(n=6)$. Other detailed information about these patients is shown in Table 1.

Tumor tissue and normal lung tissue from the resected specimens were examined during the first 15 min after resection; the tumor was localized macroscopically, and $1-\mathrm{cm}^{3}$ fragments of tumor and normal lung tissue were stored in liquid nitrogen at $-170^{\circ} \mathrm{C}$. A mirror fragment was fixed in formalin, embedded in paraffin, and stained with hematoxylin and eosin for histological evaluation, in order to guarantee the tumor representation. Two investigators (MPR and VLC) reviewed and verified the histological diagnosis in non-small-cell LC to be SqCC, AD, and LCC, classified according to the recently published classification for LC (31), and selected a sample of the paraffin-embedded tumor for immunohistochemical scoring and analyses of the cell-specific localization by immunofluorescence and confocal microscopy.

\section{Biotinylated HA probe in frozen sputum and lung tissue}

To determine the levels of HA present in the tumoral and normal lung samples collected, the tissues were dehydrated with acetone, dried, weighed, and incubated with a proteolytic enzyme for $18 \mathrm{~h}$ at $60^{\circ} \mathrm{C}$. The HA chains were diluted (1:100) in blocking buffer $(0.05 \mathrm{M}$ Tris- $\mathrm{HCl}$, $\mathrm{pH} 7.4,1 \% \mathrm{BSA})$. Determination of $\mathrm{HA}$ was performed using the same "ELISA-like" fluorometric assay as used on the sputum (32). The dry tissue samples were assayed in triplicate. The levels HA found in the tissue specimens is reported using the dry tissue weight ( $\mu \mathrm{g} / \mathrm{g}$ of dry tissue) as reference.

\section{Biotinylated HA probe and microvessels in FFPE lung tissue}

HA quantification in situ was done in FFPE sections of LC and normal tissue. All sections underwent the following treatment: a) inhibition of endogenous peroxidase activity with $3 \%$ hydrogen peroxidase in phosphatebuffered saline (PBS); $b$ ) blockage of nonspecific binding sites with $1 \%$ BSA in PBS; $c$ ) incubation in HA-biotinylated probe solution diluted $1: 600$ in $1 \%$ BSA/PBS for $1 \mathrm{~h}$ at $4{ }^{\circ} \mathrm{C} ; d$ ) elimination of endogenous biotin in tissue sections by incubation in streptavidin peroxidase solution; and $e$ ) visualization of peroxidase activity through incubation with 
chromogenic diaminobenzidine (Sigma, USA) and 0.03\% hydrogen peroxidase. Brownish staining was considered evidence of HA expression by the cells.

Microvessel evaluation was done by immunostaining using an anti-CD34 monoclonal antibody (Novocastra Laboratory, UK; dilution 1:600), pressure-cooking antigen retrieval, biotinylated rabbit anti-mouse IgG (Dako Corp., USA; dilution, 1:400), streptavidin combined in vitro with biotinylated horseradish peroxidase (Dako Corp.; dilution, 1:1000), diaminobenzidine tetrahydrochloride, and counterstaining with hematoxylin. Brownish cytoplasmic staining was considered to be evidence of the antigen expression by cells.

\section{Evaluation of HA and microvessel staining}

We evaluated the HA and microvessel staining in nonneoplastic cells, LC cells, and tumor stroma by image analysis. The HA and microvessel quantification was done by recording histological images with a Nikon Eclipse 50i camera (Japan) attached to the microscope, using a $40 \times$ objective. The staining measurements were done by computer analysis, using the Image Pro-Plus 6.0 software (Media Cybernetics, USA). All the analyses were performed at a magnification of $400 \times$.

Staining for HA was noted on the cell surface and in the cytoplasm of cancer cells and in peritumoral stroma, whereas microvessel staining was noted on endothelial cells present in tumoral and peritumoral stroma. The percentage of stained tumor parenchyma was evaluated using a continuous threshold $(0 \%-100)$. For statistical purposes, cases were further divided into two groups according to a receiver operation characteristic (ROC) curve as follows: expression of HA was considered high if $\geqslant 48 \%$ of the tumoral area showed a persistent HA signal, whereas cases having $<48 \%$ staining were classified as low. This cut-off level allowed the most clear-cut separation between high and low expressors. The intensity of HA staining in cancer cells and in peritumoral stromal tissue was classified as weak ( $<48$ and $<84 \%$, respectively, of stromal tissue/cancer cells with intense signal) or strong ( $\geqslant 48$ and $\geqslant 84 \%$, respectively, of stromal tissue/cancer cells with intense signal). Equally, the intensity of microvessel staining in tumoral stroma and peritumoral stroma was classified as weak ( $<33$ and $<26 \%$, respectively, of peritumoral stroma/tumoral stroma with intense signal) or strong ( $\geqslant 33$ and $\geqslant 26 \%$, respectively, of peritumoral stroma/tumoral stroma with intense signal).

\section{HA and microvessel colocation}

$\mathrm{HA}$ and microvessel colocation was evaluated in tumor stroma after immunofluorescence staining using confocal microscopy. Pressure-cooking antigen retrieval was performed followed by incubation with mouse polyclonal anti-human CD34 antibody (1:50; Santa Cruz Biotechnology, Inc., USA) and an HA-biotinylated probe solution (1:150; kindly donated by $\mathrm{Dr}$. Nader from the
Departamento de Bioquímica, Disciplina de Biologia Molecular, Universidade Federal de São Paulo, Brazil). For negative controls, sections were incubated with PBS instead of the primary antibody. The sections were then incubated with the secondary antibodies, i.e., goat anti-mouse ALEXA 488 and streptavidin ALEXA 546 (Invitrogen, USA; dilution 1:400) for $3 \mathrm{~h}$. Nuclear staining was done with 4',6-diamidino-2-phenylindole, dihydrochloride for 30 min (Invitrogen; 1:300). Images were obtained using a Zeiss LSM-410 laser-scanning confocal microscope (Carl Zeiss, Germany). Serial optical sections were performed with the Simple $32 \mathrm{C}$-imaging computer software (LSM Image Browser software, Carl Zeiss). Zseries sections were collected at $0.6 \mu \mathrm{m}$ with a $\times 60$ Plan Apo lens and a scan zoom of $\times 2$. All images were collected at the same photomultiplier tube settings. Images were processed and reconstructed using the US National Institutes of Health Image software.

\section{Statistical analysis}

Statistical analysis was performed using SPSS 18.0 (SPSS Inc., USA). When necessary, the variables were analyzed with the Kolmogorov-Smirnov test to determine the normality pattern. Mann-Whitney and Kruskal-Wallis tests were used to analyze the sputum from 90 patients with LC, 25 cancer-free patients, and 15 healthy volunteers. When nonparametric methods were used, simultaneous comparisons of the confidence levels were corrected with Bonferroni's post hoc test. The MantelHaenszel test or Pearson's $\chi^{2}$ test were used to clarify the relationship between $\mathrm{HA}$ and microvessel staining with the tissue and the sputum variables studied. ROC curves were developed for determining the optimal cut-off limits that yielded the best possible sensitivity and specificity values. Data on various biochemical, surgical, and pathological parameters, as well as $\mathrm{HA}$ and microvessel staining inferences, were analyzed by the Cox proportional hazards model, using single-variable analysis (univariate analysis). Stratified Kaplan-Meier analyses were performed on the variables found to be significant in the multivariate Cox proportional hazards model. The results with $\mathrm{P}$ less than or equal to 0.05 were considered to be significant.

\section{Results}

Cancer-free patients and healthy volunteers had a median age of 65 years; there was no significant difference in age between the groups. There were fewer females in the cancer-free group $(n=17)$ than in the malignant group $(n=31)$ or the LC group (Table 1$)$.

Figure 1 shows normal lung, SqCC, $A D$, and LCC stained by hematoxylin and eosin $(A, F, K, P)$, biotinylated $\mathrm{HA}$ probe $(B, G, L, Q)$, immunofluorescence double staining for $\mathrm{HA}$ and microvessel colocalization at confocal microscopy (D, I, N, S), and quantification of HA in FFPE, respectively. 
Normal lung (Figure 1A) showed a strong HA signal at the epithelial cell-extracellular matrix interface (Figure 1, B and $\mathrm{C}$ ), coincident with regular microvessel staining (C) and absence of colocalization at confocal microscopy (D). A significant increase in the HA signal was found in tumor tissue compared to normal tissue $(\mathrm{P}<0.05$; Figure $1 \mathrm{E})$.

SqCC (F) showed abundant HA signals on the cancer cell-stroma interface $(G)$ coincident with prominent microvessel staining $(\mathrm{H})$ and identical colocalization at confocal microscopy (I). Strong HA staining intensity associated with cancer cells was significant in $32.1 \%$ of SqCC compared to $17.9 \%$ of $A D$ and $0.0 \%$ in LCC ( $<<0.001$; Table 2; Figure $1 \mathrm{~J})$.

ADs (Figure $1 \mathrm{~K}$ ) revealed strong stromal HA staining in remarkable contrast with the HA-poor tumor cells in ADs (L). The foci of strong HA intensity in the stroma was coincident with foci of microvessel staining (M) and foci of colocalization at confocal microscopy $(\mathrm{N})$. Furthermore, $\mathrm{AD}$ more often showed a lower percentage of HA-positive cancer cells ( $35.7 \%$ of cases) than LCC ( $14.3 \%$ of cases) or SqCC ( $0 \%$ of cases; $\mathrm{P}<0.001$; Figure 10$)$. The cancer stroma HA staining intensity was similar among the three histological types ( $P=0.3$; Table 2).

For LCC $(P)$, the HA signal in tumor cells was very poor or absent and contrasted with the foci of strong intensity in stroma $(\mathrm{Q})$, coincident with focal microvessel density $(R)$ and focal colocalization at confocal microscopy (S). The poor qualitative distribution of HA in tumor tissue overlapped with the very low HA intensity of staining after quantitation (Figure 1T).

Table 2 shows the morphometric results for microvessels in cancer stroma and normal tissue stratified according to histological type. Microvessels were significantly higher in AD (32.3\%) and LCC (16.1\%) compared to SqCC $(3.2 \% ; P=0.02)$. No difference in microvessels was observed in normal lung stroma of the three histological types.

A significant direct association was found between tumors with a high percentage of HA and MVD in tumor
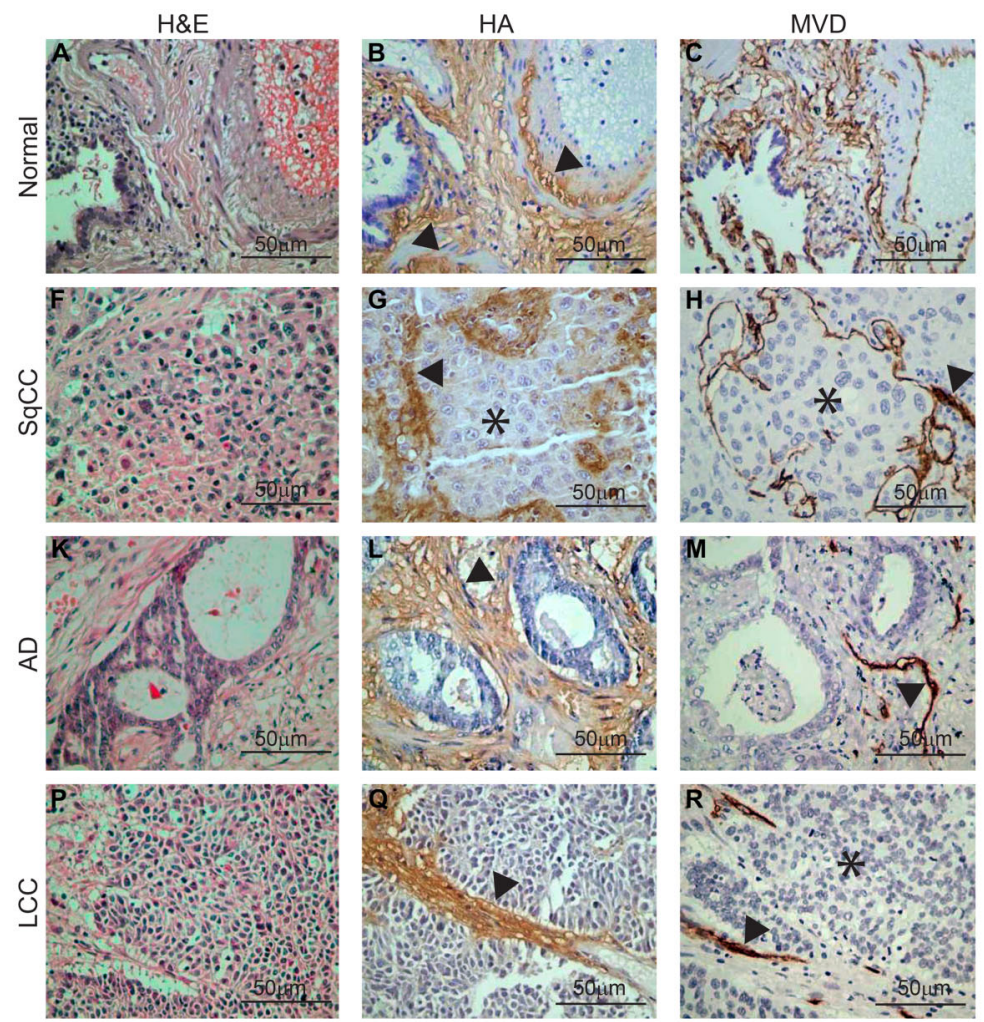
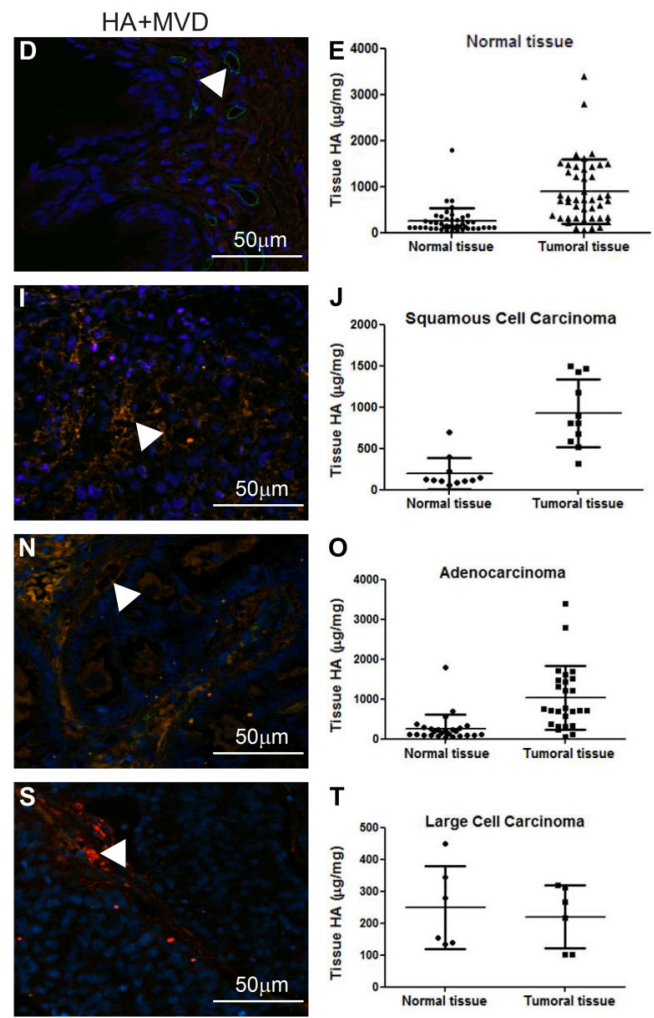

Figure 1. Strong hyaluronan $(\mathrm{HA})$ signal at the epithelial cell-extracellular matrix interface $(B)$ in normal lung $(A)$, coincident with regular microvessels staining $(C)$, absence of colocalization at confocal microscopy $(D)$ and low signal when compared to tumoral tissue $(E)$. High expression ( $\geqslant 48 \%$ ) of HA in squamous cell carcinoma (SqCC) $(F)$ on cancer cells-stroma interface $(G)$, coincident with high microvessel $(\geqslant 33 \%)$ staining foci $(H)$, identical colocalization at confocal microscopy $(I)$ and higher signal when compared to normal tissue $(J)$. HA-negative in normal lung $(K)$ and cells of adenocarcinoma (AD) (L); note the strong HA staining in tumor stroma $(L)$, coincident with microvessels staining $(M)$, foci of colocalization at confocal microscopy $(N)$ and higher expression compared to normal tissue $(O)$. Very poor signal in normal lung $(P)$ and in large cell carcinoma (LCC) $(Q)$; note strong HA foci staining $(Q)$, coincident with focal microvessels density $(R)$, focal colocalization at confocal microscopy $(S)$ and low signal when compared to normal tissue $(T)$. Arrowheads indicate stromal tissue; asterisks indicate carcinoma cells. H\&E: hematoxylin and eosin; MVD: microvessel density 
Table 2. Immunohistochemistry analysis and distribution of hyaluronan (HA) and microvessel density (MVD) in cancer cells, stroma and peritumoral stroma stratified according to histologic types of lung cancer.

\begin{tabular}{|c|c|c|c|c|}
\hline & $\begin{array}{l}\text { Squamous cell } \\
\text { carcinoma }\end{array}$ & Adenocarcinoma & Large cell & $\mathrm{P}$ \\
\hline HA in cancer cells $(\%)$ & & & & $<0.001$ \\
\hline$<48 \%$ & $0(0)$ & $10(35.7)$ & $4(14.3)$ & \\
\hline$\geqslant 48 \%$ & $9(32.1)$ & $5(17.9)$ & $0(0)$ & \\
\hline $\mathrm{HA}$ in peritumoral stroma $(\%)$ & & & & 0.3 \\
\hline$<84 \%$ & $4(14.3)$ & $7(25)$ & $3(10.7)$ & \\
\hline$\geqslant 84 \%$ & $5(17.9)$ & $8(28.6)$ & $1(3.6)$ & \\
\hline MVD in tumoral stroma (\%) & & & & 0.02 \\
\hline$<33 \%$ & $6(19.4)$ & $8(25.8)$ & $1(3.2)$ & \\
\hline$\geqslant 33 \%$ & $1(3.2)$ & $10(32.3)$ & $5(16.1)$ & \\
\hline MVD in peritumoral stroma (\%) & & & & 0.5 \\
\hline$<26 \%$ & $4(14.3)$ & $8(28.6)$ & $1(3.6)$ & \\
\hline$\geqslant 26 \%$ & $3(10.7)$ & $9(32.1)$ & $3(10.7)$ & \\
\hline
\end{tabular}

Data are reported as the number of cases with percent in parentheses. Groups were divided as high $(\geqslant 48 \%)$ and low $(<48 \%)$ according to the best cut-off determined by ROC curve. The Pearson $\chi^{2}$ test $(\mathrm{P}<0.05)$ was used for statistical analyses.

stroma $(R=0.6 ; P=0.02)$. Similarly significant was the direct association between tumors at the $\mathrm{N} 1$ stage and high levels of $H A$ in cancer cells $(R=0.31 ; P=0.05)$. In addition, tumors in the T4 stage presented positive association with a high percentage of HA-positive cancer cells $(R=0.80 ; P=0.01)$.

At the end of the follow-up, 32 patients of $46(69.5 \%)$ who underwent surgical resection died because of LC. Univariate survival analysis showed that the significant predictors of overall survival (OS) were $\mathrm{N}$ stage $\left(\chi^{2}=4.3\right.$; $\mathrm{P}=0.03)$ and $\mathrm{HA}$ expression in cancer cells $\left(\chi^{2}=5.2\right.$; $\mathrm{P}=0.02)$. A high percentage of HA-positive cells predicted a shortened disease-free survival $[n=11(47.8 \%)$, $\mathrm{P}=0.02]$ in univariate analysis together with stage $(P=0.02)$. However, among ADs, there was a trend toward poor OS, with tumors showing a high percentage of cancer cell-associated HA $(P=0.09)$. Comparative Cox multivariate analysis controlled by gender, age, and $A D$ showed a significant association between better survival and low cancer cell-associated $\mathrm{HA}$ sign $(\mathrm{HR}=0.08$; $P=0.03$ ). We ranked the cases according to ROC curve into two groups with distinctly different average survival times as illustrated by Kaplan-Meier plots in Figure 3. The group with $<692.1 \mu \mathrm{g} / \mathrm{mg} \mathrm{HA}$ appears as the top curve, and their median survival time was 72 months. Those with $\geqslant 692.1 \mu \mathrm{g} / \mathrm{mg} \mathrm{HA}$ (bottom curve) had a median survival time of just 52 months after surgery (log rank $=5.3$; $\mathrm{P}=0.02$ ).

Table 2 shows the concentration levels of HA found in normal and cancer lung tissue surgically resected from 46 patients with LC. HA concentration levels were significantly increased in tissue from patients with LC compared to normal lung tissue $(P<0.001)$. HA concentration levels increased seven-fold in cancer tissue compared to normal lung tissue. Concentration levels of HA were significantly higher in lung tissue from patients with SqCC than $A D$ and LCC ( $P=0.01$; Table 2), coincident with the qualitative and quantitative immunoexpression observed in FFPE (Figure 1, G and J).

The concentration levels of HA in sputum obtained from 90 patients with LC, 25 cancer-free patients, and 15 healthy volunteers are shown in Table 3. HA concentration levels were significantly increased in sputum from LC patients compared to cancer-free and healthy volunteers $(P<0.001$, Figure 2A). HA levels were higher in the sputum of patients with SqCC compared to other histological types ( $P=0.01$; Table 3 , Figure $2 B)$.

The sputum HA analysis capacity to distinguish healthy volunteers from LC patients was analyzed using an ROC curve. The area under the curve for this condition was $0.821(0.727-0.915)$. Assuming a cut-off value of $20.06 \mathrm{ng} / \mathrm{mg}$, the specificity was $80 \%$ and the sensitivity was $66 \%$ (Figure $2 \mathrm{C}$ ). The sputum $\mathrm{HA}$ analysis capacity to distinguish cancer-free patients from LC patients was analyzed by a second ROC curve. The area under the curve for this condition was $0.698(0.600-0.797)$, and the cut-off value of $24.04 \mathrm{ng} / \mathrm{mg}$ showed $68 \%$ specificity and $60 \%$ sensitivity (Figure 2D).

A significant correlation was found between the biotinylated $\mathrm{HA}$ probe in sputum and biotinylated $\mathrm{HA}$ FFPE cancer tissue $(R=0.5 ; P=0.02)$.

\section{Discussion}

The first aim of this research was to evaluate the association between malignant changes and HA through its expression and location in tumor tissue and its 

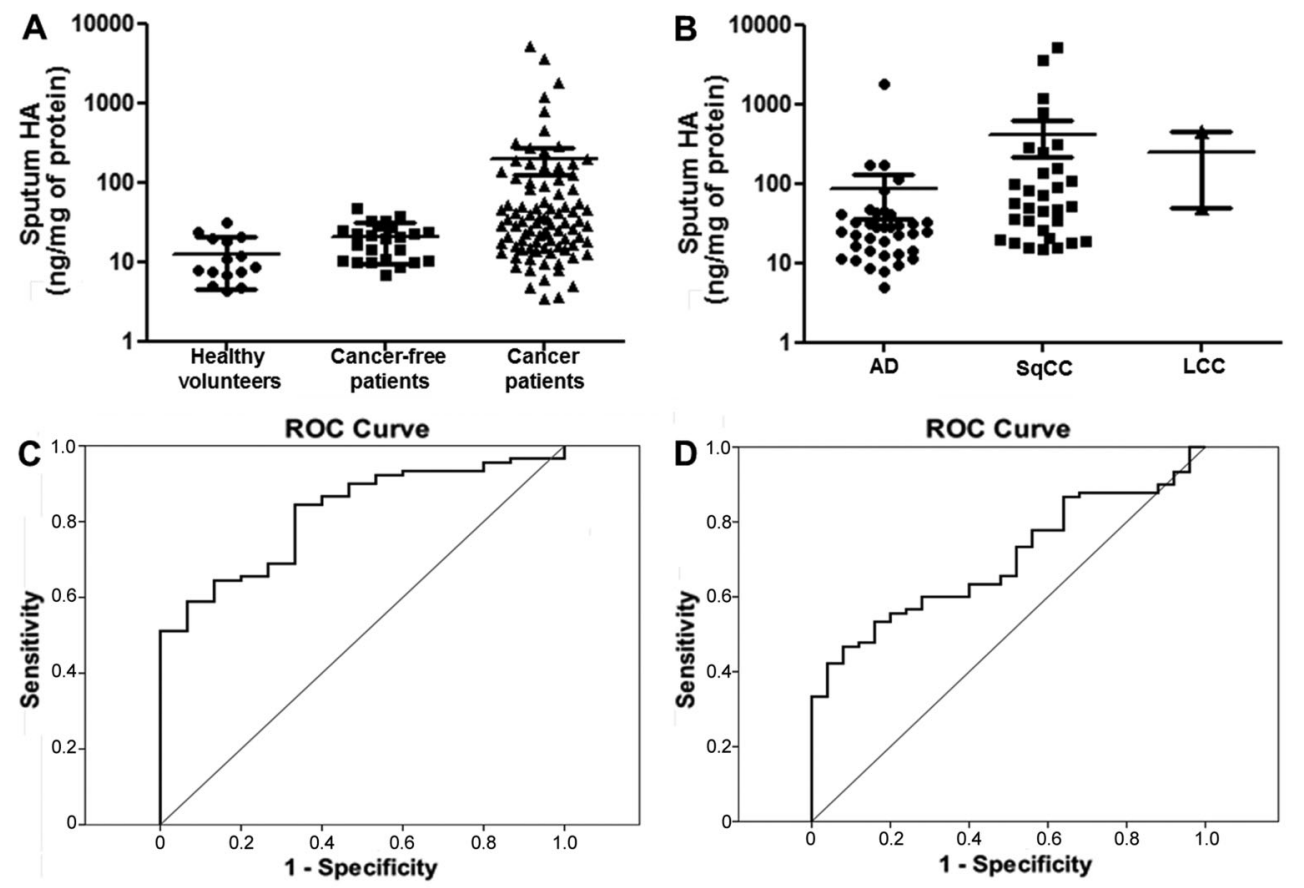

Figure 2. $A$, Box plots of hyaluronan $(\mathrm{HA})$ in healthy volunteers, cancer-free patients and lung cancer patients (LC). Patients with lung cancer had significantly higher HA levels in sputum than in the cancer-free patients and healthy volunteers (Log10 scale; $\mathrm{P}<0.001$, Mann-Whitney test). $B$, Box plots of HA in lung cancer patients. Patients with squamous cell carcinoma (SqCC) had higher HA levels compared to adenocarcinoma (AD) and large cell carcinoma (LCC) (log10 scale; $\mathrm{P}<0.001$, Kruskal-Wallis test). C, D, Receiver operating characteristic (ROC) curves for sputum levels of HA. C, The cut-off level of HA that resulted in the highest diagnostic accuracy was $>31.44 \mathrm{ng} / \mathrm{mg}$. This cut-off point discriminated between healthy controls and lung cancer patients, with $100 \%$ specificity and $51 \%$ sensitivity. The diagnostic accuracy was $82 \%$. D, The cut-off level of HA that discriminated cancer-free patients and lung cancer patients with $100 \%$ specificity and $33 \%$ sensitivity was $>48.3 \mathrm{ng} / \mathrm{mg}$. The diagnostic accuracy was $69 \%$.

relationship with histological types, angiogenesis, and metastases. Hypothesizing to get an association between $\mathrm{HA}$ and malignant changes on prognosis, the second aim was to evaluate whether these malignant changes were reflected in sputum from patients with $L C$.

We found a significant expression of HA and microvessels at the epithelial cell-extracellular matrix interface in tumoral lung tissue, and confocal microscopy confirmed this colocalization, suggesting that an increased synthesis of HA occurs through interactions with $\mathrm{HA}$ receptors and endothelium. HA receptors are expressed by endothelial cells (33), lung fibroblasts, and smooth muscle (34), which are abundant at the cell-extracellular matrix interface. This coupled expression of $\mathrm{HA}$ receptors and $\mathrm{HA}$ at the epithelial cell-extracellular matrix interface suggested that these two components must interact with each other. This interaction could play a role in attaching respiratory epithelial cells to the basement membrane and in maintaining their polarized morphology. When the malignant transformation occurs in respiratory epithelial cells, the imbalance between hyperexpression of $\mathrm{HA}$ and $\mathrm{HA}$ synthases (14) is unable to attach respiratory epithelial cells to the basement membrane, leading to invasion and exfoliation in fluids, such as sputum. The direct association that we found between a high percentage of HA and microvessels in the tumor cell-stroma interface is supported by the concept that hyaluronidases (HYAL1 and HYAL2), which are responsible for HA degradation (14), stimulate malignant neovascularization (15) for tumor growth and metastases. Indeed, when we analyzed the entire tumor, we found a direct association between tumors in T4 and N1 stages and high levels of HA in cancer cells, clearly suggesting that surgical resection fails to cure some patients with localized LC because of metastases.

In the current study, a strong HA staining intensity associated with cancer cells was also significant in SqCCs compared to AD and LCC. Similar findings were reported by Pirinen et al. (25), who analyzed the prognostic value of $\mathrm{HA}$ in a large series of patients with non-small-cell lung carcinoma using a biotinylated HA-specific probe. As previously reported by Wang et al. (27), tumor cells were enhanced in $\mathrm{HA}$, but they showed considerable differences in HA expression depending on the cellular origin as well as on the histological type of the tumor. Our findings also support the concept that normal squamous 


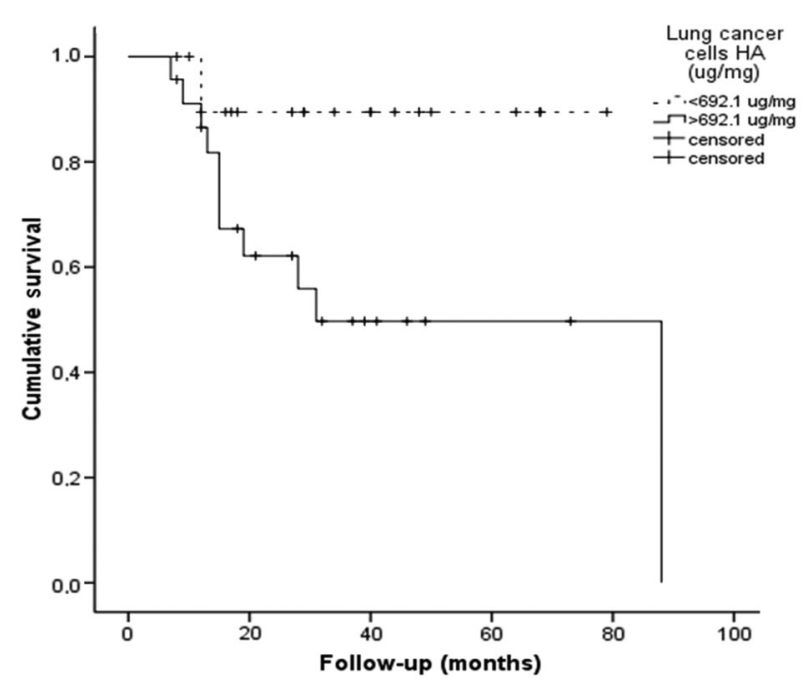

Figure 3. Kaplan-Meier plots of survival probability vs follow-up time in months for all patients. The group with $<692.1 \mu \mathrm{g} / \mathrm{mg} \mathrm{HA}$ appears as the top curve, and the group with $\geqslant 692.1 \mu \mathrm{g} / \mathrm{mg} \mathrm{HA}$ appears as the bottom curve.

cells and carcinomas of squamous cell differentiation mostly express large amounts of HA (29). In contrast, tumors derived from nonstratified epithelium, such as colon (28), ovarian (35), and breast (32) tumors, show an absence of or a very poor HA signal. In agreement with these reports, our present results showed a relatively low percentage of high HA expression in AD. Likewise, our findings showed that an elevated HA signal in tumor cells was associated with poor prognosis, similar to results in the literature $(25,28,32,35)$.

Thus, we should not be surprised to learn that a biotinylated HA-specific probe provided important prognostic information about cancerous changes in tissue, and our results now confirm the prognostic importance of HA in LC. Whereas only four prior studies were able to show a significant relationship between immunostaining for $\mathrm{HA}$ in the tumor and survival $(25,28,32,35)$, our results suggest that staining for $\mathrm{HA}$, used as a continuous variable, provides more prognostic information than does routine pathological stage. A binary cut-off point in HA in tumors was the median of $692.1 \mu \mathrm{g} / \mathrm{mg}$ of cells staining for HA, and this point provided a practical way to separate patients into two groups: patients with an expected short survival vs patients with an expected longer survival. Thus, a biotinylated HA-specific probe for HA in the primary tumor offered the potential to show malignant changes in FFPE tissue from patients with LC.

The next question of interest was whether these malignant changes gathered from the tumor tissue or its milieu could help us identify patients with LC using a noninvasive procedure such as sputum. We found that HA concentration levels were significantly increased in sputum from LC patients compared to cancer-free and healthy volunteers. One of the mechanisms by which HA increases in sputum may be via the promotion of cell migration, because of the highly expanded, hydrated extracellular space provided by $\mathrm{HA}$, which facilitates cell movement, and by $\mathrm{HA}$ signaling through its receptors CD44 and RHAMM (36), as shown in previous studies $(34,36)$. As already discussed, malignant changes induce a disproportion between expression of $\mathrm{HA}$ and hyaluronidase by respiratory epithelial cells (14), which become unable to attach cells to the basement membrane, leading to exfoliation and hypersecretion of HA in sputum. Both events lead to detachment of the HA layer that usually covers glands and the respiratory epithelium (14). There is another possible mechanism by which HA is deposited in sputum. Malignant cells affect tumoral stroma remodeling by regulating fibroblasts. The activated stromal

Table 3. Tissue and sputum hyaluronan (HA) analysis.

\begin{tabular}{lcc}
\hline & Tissue $(\mu \mathrm{g} / \mathrm{g})$ & Sputum $(\mathrm{ng} / \mathrm{mg})$ \\
\hline Lung cancer & $713.13 \pm 104.55^{\star}$ & \\
Squamous cell carcinoma & $808.28 \pm 222.03$ & \\
Adenocarcinoma & $717.97 \pm 135.28$ & \\
Large cell carcinoma & $241.7 \pm 40.14$ & \\
Normal lung & $155.39 \pm 39.28$ & $31.72 \pm 2.97^{+}$ \\
Lung cancer & & $19.78 \pm 2.21$ \\
Cancer-free patients & & $8.72 \pm 2.11$ \\
Healthy volunteers & & \\
\hline
\end{tabular}

Data are reported as median \pm SE of HA in lung specimens from tumors and sputum. In both specimens, determination of HA was performed using the same "ELISA-like" fluorometric assay. ${ }^{*} \mathrm{P}<0.001$, HA levels in lung cancer tissue specimens were significantly different from normal lung tissue and maintained when categorized by histological types $(\mathrm{P}<0.001$ and $\mathrm{P}=0.005) .{ }^{+} \mathrm{P}<0.001$, HA levels in sputum of lung cancer patients were significantly different from cancer-free patients and healthy subjects. The Mann-Whitney test was used for statistical analyses. 
fibroblasts, termed cancer-associated fibroblasts (CAFs), share some similarities with myofibroblasts, including the expression of alpha smooth muscle actin (37). CAFs play a prominent role in the remodeling process by producing collagen and elastin fibers as well as proteoglycans and glycoproteins (38). It has also been shown that airway fibroblasts from LC patients produce significantly increased concentrations of $\mathrm{HA}$ and $\mathrm{HA}$ synthase compared to controls $(4,5)$. LC tumors secrete tumor necrosis factor alpha (TNF- $\alpha$ ), tumor growth factor beta, and endothelial growth factor, known to be potent stimulators of HA synthesis in lung fibroblasts, and may contribute to the deposition of HA in the airways of LC. In addition, tumors express CD44, which binds $\mathrm{HA}$, and this interaction has been implicated in aggregation, proliferation, and migration of neoplastic cells $(24,25,34)$. Therefore, it is possible that LC patients express a pathological feedback loop where tumors stimulate fibroblast synthesis of $\mathrm{HA}$ through the release of tumor growth factor beta and TNF- $\alpha$, and increase recruitment of additional fibroblasts through upregulation of CD44.

We also found that HA levels were higher in the sputum of patients with SqCC compared to other histological types of tumor. SqCC carcinogenesis depends on epigenetic factors, and it has already been reported that the connection between HA and its receptor CD44 can result in the suppression of several epigenetic regulators and the upregulation of survival proteins. In addition, the greater quantities of $\mathrm{HA}$ in the SqCC cancer cells were reflected in the greater quantities of $\mathrm{HA}$ found in the sputum from SqCC patients in this study, and that might be explained by the central bronchial location and squamous cell origin of the tumor, whereas $A D$ and LCC are located peripherally and originate from columnar gland cells.

Previous studies have demonstrated that $\mathrm{HA}$ is elevated in malignant mesothelioma effusions. In this context, Pettersson et al. (17) found that, with the cut-off level at $100 \mathrm{mg} / \mathrm{L}$, the pleural fluid concentration of HA was increased in $73 \%$ of patients with mesothelioma and $23 \%$ of patients with nonmalignant inflammatory diseases, but in none of the patients with cancer other than mesothelioma and in none of the patients with congestive heart failure. Other studies have demonstrated that HA is elevated in urine from patients with urologic cancer. Lokeshwar et al. (39) compared levels of $\mathrm{HA}$ in the urine of normal individuals and patients with bladder cancer or other genitourinary conditions, and found that the urinary HA measurement by the ELISA-like assay shows a sensitivity of $91.9 \%$ and specificity of $92.8 \%$ to detect bladder cancer. In inflammatory conditions, such as chronic obstructive pulmonary disease and asthma, HA has also been applied for diagnostic purposes in sputum. Ayars et al. (30) examined levels of HA in the sputum of asthmatics treated with mepolizumab therapy vs those taking a placebo, and found reduced levels of $\mathrm{HA}$ as well as improved clinical and spirometry values in the patients treated with mepolizumab, suggesting that this measurement may serve as a noninvasive biomarker of asthma control.

To our knowledge, the current study is the first to investigate $\mathrm{HA}$ as an indicator in the sputum from patients with LC, therefore limiting a comparison of the results, unless they are compared to the current gold standard, sputum cytology. Presently, sputum cytology is the minimum invasive resource for obtaining a diagnosis in a patient who is suspected of having LC. However, the diagnostic accuracy of sputum cytology depends on rigorous specimen sampling and preservation techniques, as well as on the location and size of the tumor (central vs peripheral). In the present study, we showed that, using a single low-cost procedure that is not time consuming and that can be performed routinely in the lab, sputum HA distinguished tumor patients from those without tumor with $80 \%$ specificity and $66 \%$ sensitivity. Similarly, the sputum HA concentration can distinguish cancer-free patients from LC patients with $68 \%$ specificity and $60 \%$ sensitivity. According to the literature, sputum cytology sensitivity ranges from $42 \%$ to $97 \%$ and specificity ranges from 68 to $100 \%$ (40). Our findings contrast with a recent study published by Van Rensburg et al. (31) in which the sputum cytology in a high-risk population revealed a sensitivity of $38.9 \%$ to detect malignant cells and $100 \%$ accuracy. Comparing our findings to the literature, we presume that the sensitivities between $60 \%$ and $66 \%$ that we found can be within the same limits of variation as cytology sputum. Therefore, HA evaluation in sputum could be superior or similar to the current gold standard, sputum cytology, eventually emphasizing that combined sputum HA and cytology may add considerably to the diagnostic armamentarium currently used in the clinic for LC diagnosis.

Localization of HA in tumor tissue and its relationship with histological types and angiogenesis was related to malignancy of LC and reflected in sputum, which is emerging as an important diagnostic procedure in patients with suspicion of LC. To confirm these results, more studies in a randomized and prospective trial are needed, and we also believe it is important to validate our quantitative assessment of $\mathrm{HA}$ and to couple it to gold standard sputum cytology by studying HA in additional patients.

\section{Acknowledgments}

We are grateful to Biologist Sandra de Morais Fernezlian, Laboratory of Immunohistochemistry for her technical assistance. Research supported by FAPESP (2010/11005-5 and 2010/04462) and CNPq (\#471939/ 2010-2 and 483005/2012-6). Oral Presentation at the 2011 and 2012 European Respiratory Congress (Silver Sponsorship). 


\section{References}

1. Schrump D, Altorki N, Henschke C, Carter D, Turrisi A, Gutierrez M. Non-small cell lung cancer. In: De Vita V, Hellman S, Rosenberg S (Editors), Cancer: principles and practice of oncology. 7th edn. Philadelphia: Lippincott Williams \& Wilkins; 2005. p 753-754.

2. Howlader N, Noone AM, Krapcho M, Neyman N, Aminou R, Waldron W, et al. SEER Cancer Statistics Review, 19752008. http://seer.cancer.gov/archive/csr/1975_2008/. Accessed December 26, 2011.

3. Wistuba II, Gazdar AF. Lung cancer preneoplasia. Annu Rev Pathol 2006; 1: 331-348, doi: 10.1146/annurev.pathol.1. 110304.100103

4. de Sá VK, Carvalho L, Gomes A, Alarcão A, Silva MR, Couceiro $P$, et al. Role of the extracellular matrix in variations of invasive pathways in lung cancers. Braz $J$ Med Biol Res 2013; 46: 21-31, doi: 10.1590/1414-431X20 122263.

5. de Sa V, Olivieri E, Parra ER, Ab'Saber AM, Takagaki T, Soares FA, et al. Hyaluronidase splice variants are associated with histology and outcome in adenocarcinoma and squamous cell carcinoma of the lung. Hum Pathol 2012; 43: 675-683, doi: 10.1016/j.humpath.2011.06.010.

6. Souza P, Rizzardi F, Noleto G, Atanazio M, Bianchi O, Parra ER, et al. Refractory remodeling of the microenvironment by abnormal type $\mathrm{V}$ collagen, apoptosis, and immune response in non-small cell lung cancer. Hum Pathol 2010; 41: 239-248, doi: 10.1016/j.humpath.2009.07.018.

7. Zetter BR. Adhesion molecules in tumor metastasis. Semin Cancer Biol 1993; 4: 219-229.

8. Freemont $\mathrm{T}$. The significance of adhesion molecules in diagnostic histopathology. Curr Diag Pathol 1995; 2: 101110, doi: 10.1016/S0968-6053(00)80006-X.

9. Yang C, Cao M, Liu H, He Y, Xu J, Du Y, et al. The high and low molecular weight forms of hyaluronan have distinct effects on CD44 clustering. J Biol Chem 2012; 287: 4309443107, doi: 10.1074/jbc.M112.349209.

10. Papakonstantinou E, Karakiulakis G, Eickelberg O, Perruchoud AP, Block LH, Roth M. A 340 kDa hyaluronic acid secreted by human vascular smooth muscle cells regulates their proliferation and migration. Glycobiology 1998; 8: 821-830, doi: 10.1093/glycob/8.8.821.

11. Toole BP. Glycosaminoglycans and morphogenesis. In: Hay ED (Editor), Cell biology and extracellular matrix. New York: Plenum Press; 1991. p 259-294.

12. Toole BP. Hyaluronan: from extracellular glue to pericellular cue. Nat Rev Cancer 2004; 4: 528-539, doi: 10.1038/nrc1391.

13. Itano N, Sawai T, Yoshida M, Lenas $P$, Yamada Y, Imagawa $M$, et al. Three isoforms of mammalian hyaluronan synthases have distinct enzymatic properties. $\mathrm{J} \mathrm{Biol} \mathrm{Chem}$ 1999; 274: 25085-25092, doi: 10.1074/jbc.274.35.25085.

14. Dentener MA, Vernooy JH, Hendriks S, Wouters EF. Enhanced levels of hyaluronan in lungs of patients with COPD: relationship with lung function and local inflammation. Thorax 2005; 60: 114-119, doi: 10.1136/thx.2003. 020842.

15. Deed R, Rooney P, Kumar P, Norton JD, Smith J, Freemont $\mathrm{AJ}$, et al. Early-response gene signalling is induced by angiogenic oligosaccharides of hyaluronan in endothelial cells. Inhibition by non-angiogenic, high-molecular-weight hyaluronan. Int J Cancer 1997; 71: 251-256, doi: 10.1002/ (SICI)1097-0215(19970410)71:2<251::AID IJC21>3.0.CO;2-J.

16. Roboz J, Greaves J, Silides D, Chahinian AP, Holland JF. Hyaluronic acid content of effusions as a diagnostic aid for malignant mesothelioma. Cancer Res 1985; 45: 1850-1854.

17. Pettersson $T$, Froseth $B$, Riska $H$, Klockars $M$. Concentration of hyaluronic acid in pleural fluid as a diagnostic aid for malignant mesothelioma. Chest 1988; 94: 1037-1039, doi: 10.1378/chest.94.5.1037.

18. Atagi S, Ogawara M, Kawahara M, Sakatani M, Furuse K, Ueda $\mathrm{E}$, et al. Utility of hyaluronic acid in pleural fluid for differential diagnosis of pleural effusions: likelihood ratios for malignant mesothelioma. Jpn J Clin Oncol 1997; 27: 293297, doi: 10.1093/jjco/27.5.293.

19. Welker L, Muller M, Holz O, Vollmer E, Magnussen H, Jorres RA. Cytological diagnosis of malignant mesothelioma--improvement by additional analysis of hyaluronic acid in pleural effusions. Virchows Arch 2007; 450: 455-461, doi: 10.1007/s00428-007-0375-x.

20. Creaney J, Dick IM, Segal A, Musk AW, Robinson BW. Pleural effusion hyaluronic acid as a prognostic marker in pleural malignant mesothelioma. Lung Cancer 2013; 82 491-498, doi: 10.1016/j.lungcan.2013.09.016.

21. Nossier Al, Eissa S, Ismail MF, Hamdy MA, Azzazy HM. Direct detection of hyaluronidase in urine using cationic gold nanoparticles: a potential diagnostic test for bladder cancer. Biosens Bioelectron 2014; 54: 7-14, doi: 10.1016/j.bios. 2013.10.024.

22. Kramer MW, Escudero DO, Lokeshwar SD, Golshani R, Ekwenna OO, Acosta K, et al. Association of hyaluronic acid family members (HAS1, HAS2, and HYAL-1) with bladder cancer diagnosis and prognosis. Cancer 2011; 117: 11971209, doi: 10.1002/cncr.25565.

23. Passerotti CC, Srougi M, Bomfim AC, Martins JR, Leite KR, Dos Reis ST, et al. Testing for urinary hyaluronate improves detection and grading of transitional cell carcinoma. Urol Oncol 2011; 29: 710-715, doi: 10.1016/j.urolonc.2009. 10.006 .

24. Passerotti CC, Bonfim A, Martins JR, Dall'Oglio MF, Sampaio LO, Mendes A, et al. Urinary hyaluronan as a marker for the presence of residual transitional cell carcinoma of the urinary bladder. Eur Urol 2006; 49: 7175, doi: 10.1016/j.eururo.2005.09.015.

25. Pirinen R, Tammi R, Tammi M, Hirvikoski P, Parkkinen JJ, Johansson R, et al. Prognostic value of hyaluronan expression in non-small-cell lung cancer: Increased stromal expression indicates unfavorable outcome in patients with adenocarcinoma. Int J Cancer 2001; 95: 12-17, doi: 10.1002/ 1097-0215(20010120)95:1<12::AID-IJC1002>3.0.CO;2-E.

26. Pirinen RT, Tammi RH, Tammi MI, Paakko PK, Parkkinen $\mathrm{JJ}$, Agren UM, et al. Expression of hyaluronan in normal and dysplastic bronchial epithelium and in squamous cell carcinoma of the lung. Int $J$ Cancer 1998; 79: 251-255, doi: $10.1002 /(\mathrm{SICl}) 1097-0215(19980619) 79: 3<251:: A I D-$ IJC7>3.0.CO;2-O.

27. Wang $\mathrm{C}$, Tammi M, Guo H, Tammi R. Hyaluronan distribution in the normal epithelium of esophagus, stomach, and colon and their cancers. Am J Pathol 1996; 148: 1861-1869. 
28. Ropponen K, Tammi M, Parkkinen J, Eskelinen M, Tammi $\mathrm{R}$, Lipponen $\mathrm{P}$, et al. Tumor cell-associated hyaluronan as an unfavorable prognostic factor in colorectal cancer. Cancer Res 1998; 58: 342-347.

29. Hirvikoski $P$, Tammi R, Kumpulainen E, Virtaniemi J, Parkkinen JJ, Tammi M, et al. Irregular expression of hyaluronan and its CD44 receptor is associated with metastatic phenotype in laryngeal squamous cell carcinoma. Virchows Arch 1999; 434: 37-44, doi: 10.1007/ s004280050302.

30. Ayars AG, Altman LC, Potter-Perigo S, Radford K, Wight TN, Nair P. Sputum hyaluronan and versican in severe eosinophilic asthma. Int Arch Allergy Immunol 2013; 161: 65-73, doi: $10.1159 / 000343031$.

31. Van Rensburg A, Neethling GS, Schubert PT, Koegelenberg CF, Wright CA, Bolliger CT, et al. Impact of routine sputum cytology in a population at high risk for bronchial carcinoma. Int J Tuberc Lung Dis 2014; 18: 607612, doi: 10.5588/ijtld.13.0806.

32. Martins JR, Passerotti CC, Maciel RM, Sampaio LS, Dietrich CP, Nader HB. Practical determination of hyaluronan by a new noncompetitive fluorescence-based assay on serum of normal and cirrhotic patients. Anal Biochem 2003; 319: 65-72, doi: 10.1016/S0003-2697(03)00251-3.

33. Antonelli A, D'Amore PA. Density-dependent expression of hyaluronic acid binding to vascular cells in vitro. Microvasc Res 1991; 41: 239-251, doi: 10.1016/0026-2862(91)90025-7.
34. Hall CL, Yang B, Yang X, Zhang S, Turley M, Samuel S, et al. Overexpression of the hyaluronan receptor RHAMM is transforming and is also required for $\mathrm{H}$-ras transformation. Cell 1995; 82: 19-26, doi: 10.1016/0092-8674(95)90048-9.

35. Anttila MA, Tammi RH, Tammi MI, Syrjanen KJ, Saarikoski SV, Kosma VM. High levels of stromal hyaluronan predict poor disease outcome in epithelial ovarian cancer. Cancer Res 2000; 60: 150-155.

36. Knudson W. Tumor-associated hyaluronan. Providing an extracellular matrix that facilitates invasion. $A m \mathrm{~J}$ Pathol 1996; 148: 1721-1726.

37. Cirri $P$, Chiarugi $P$. Cancer associated fibroblasts: the dark side of the coin. Am J Cancer Res 2011; 1: 482-497.

38. Mantoni TS, Lunardi S, Al-Assar O, Masamune A, Brunner TB. Pancreatic stellate cells radioprotect pancreatic cancer cells through beta1-integrin signaling. Cancer Res 2011; 71: 3453-3458, doi: 10.1158/0008-5472.CAN-10-1633.

39. Lokeshwar VB, Obek C, Soloway MS, Block NL. Tumorassociated hyaluronic acid: a new sensitive and specific urine marker for bladder cancer. Cancer Res 1997; 57: 773777.

40. Rivera MP, Mehta AC, Wahidi MM. Establishing the diagnosis of lung cancer: Diagnosis and management of lung cancer, 3rd edn.: American College of Chest Physicians evidence-based clinical practice guidelines. Chest 2013; 143: e142S-e165S, doi: 10.1378/chest.122353. 